\title{
Preterm birth and risk of type 1 and type 2 diabetes: a national cohort study
}

\author{
Casey Crump ${ }^{1,2} \cdot$ Jan Sundquist ${ }^{1,2,3} \cdot$ Kristina Sundquist ${ }^{1,2,3}$
}

Received: 30 July 2019 / Accepted: 16 October 2019 / Published online: 5 December 2019

(C) The Author(s) 2019

\begin{abstract}
Aims/hypothesis Preterm birth (gestational age $<37$ weeks) has been associated with insulin resistance early in life. However, no large population-based studies have examined risks of type 1 and type 2 diabetes and potential sex-specific differences from childhood into adulthood. Clinicians will increasingly encounter adults who were born prematurely and will need to understand their long-term risks. We hypothesised that preterm birth is associated with increased risks of type 1 and type 2 diabetes into adulthood.

Methods A national cohort study was conducted of all 4,193,069 singletons born in Sweden during 1973-2014, who were followed up for type 1 and type 2 diabetes identified from nationwide diagnoses and pharmacy data to the end of 2015 (maximum age 43 years; median age at the end of follow-up 22.5 years). Cox regression was used to adjust for potential confounders, and cosibling analyses assessed the influence of shared familial (genetic and/or environmental) factors.

Results In 92.3 million person-years of follow-up, 27,512 (0.7\%) and $5525(0.1 \%)$ people were identified with type 1 and type 2 diabetes, respectively. Gestational age at birth was inversely associated with both type 1 and type 2 diabetes risk. Adjusted HRs for type 1 and type 2 diabetes at age <18 years associated with preterm birth were $1.21(95 \% \mathrm{CI}, 1.14,1.28)$ and 1.26 (95\% CI, 1.01 , 1.58), respectively, and at age 18-43 years were 1.24 (95\% CI, 1.13, 1.37) and 1.49 (95\% CI, 1.31, 1.68), respectively, compared with full-term birth. The associations between preterm birth and type 2 (but not type 1) diabetes were stronger among females (e.g. at age 18-43 years, females: adjusted HR, 1.75; 95\% CI, 1.47, 2.09; males: 1.28; 95\% CI, 1.08, 1.53; $p<0.01$ for additive and multiplicative interaction). These associations were only partially explained by shared genetic or environmental factors in families. Conclusions/interpretation In this large national cohort, preterm birth was associated with increased risk of type 1 and type 2 diabetes from childhood into early to mid-adulthood. Preterm-born children and adults may need early preventive evaluation and long-term monitoring for diabetes.
\end{abstract}

Keywords Adult $\cdot$ Diabetes mellitus, type $1 \cdot$ Diabetes mellitus, type $2 \cdot$ Gestational age $\cdot$ Infant, small for gestational age Premature birth · Preterm birth

Electronic supplementary material The online version of this article (https://doi.org/10.1007/s00125-019-05044-z) contains peer-reviewed but unedited supplementary material, which is available to authorised users.

Casey Crump

casey.crump@mssm.edu

$\triangle$ Kristina Sundquist

Kristina.Sundquist@med.lu.se

1 Department of Family Medicine and Community Health, Icahn School of Medicine at Mount Sinai, One Gustave L. Levy Place, Box 1077, New York, NY 10029, USA

2 Department of Population Health Science and Policy, Icahn School of Medicine at Mount Sinai, New York, NY, USA

3 Center for Primary Health Care Research, Lund University, Clinical Research Centre (CRC), building 28, floor 11, Jan Waldenströms gata 35, Skåne University Hospital, SE-205 02 Malmö, Sweden

\section{Abbreviations}

AGA Appropriate for gestational age

LGA Large for gestational age

SGA Small for gestational age

\section{Introduction}

Diabetes is increasing in prevalence and now affects nearly 1 in 10 adults in the US [1] and worldwide [2]. In addition to family history and unhealthy lifestyle factors, early life exposures have been identified as potential risk factors for the development of diabetes later in life. According to Barker's developmental origins theory, intrauterine nutritional 


\section{Research in context}

\section{What is already known about this subject?}

- Preterm birth has previously been associated with insulin resistance early in life

- No large cohort studies have examined the risks of type 1 and type 2 diabetes and potential sex-specific differences from childhood into adulthood

- Because of the high prevalence and improved treatment of preterm birth, clinicians will increasingly encounter individuals born prematurely and will need to understand their long-term risks

\section{What is the key question?}

- Is preterm birth associated with increased risk of type 1 and type 2 diabetes into adulthood?

What are the new findings?

- In a population-based cohort of over 4 million people, preterm birth (gestational age $<37$ weeks) was associated with approximately 1.2- and 1.3-fold risk of type 1 and type 2 diabetes, respectively, at age $<18$ years, and 1.2- and 1.5 -fold risk, respectively, at age 18-43 years

- The associations between preterm birth and type 2 (but not type 1) diabetes were significantly stronger among females

- These findings were only partially explained by shared genetic or environmental factors in families

How might this impact on clinical practice in the foreseeable future?

- Preterm-born children and adults may need early preventive evaluation and long-term monitoring for the development of type 1 or type 2 diabetes

abnormalities may permanently alter the body's structure and metabolism, resulting in early life programming for future development of diabetes and other cardiometabolic disorders [3]. Recent evidence has suggested that developmental programming on the background of preterm birth may be particularly important for future outcomes [4-6]. Approximately $11 \%$ of all births worldwide [7] and $10 \%$ in the US $[8,9]$ occur preterm (gestational age $<37$ weeks), and $>95 \%$ of preterm infants in developed countries now survive into adulthood [6]. As a result, large numbers of preterm survivors (e.g. $>400,000 /$ year in the US) are now reaching adulthood (age $\geq 18$ ) each year. Clinicians will increasingly encounter adult patients who were born preterm and will need to understand their long-term diabetes risks to guide preventive actions and anticipatory screening across the life course.

The largest prior studies of preterm birth and diabetes have focused on childhood (age $<15$ years) and reported increased risks of type 1 diabetes in preterm-born children [10-15]. A few smaller studies have also reported associations with type 2 diabetes in mid-adulthood [16-19]. However, to our knowledge, no large cohort studies have examined the risks of type 1 and type 2 diabetes and potential sex-specific differences from childhood into adulthood. Furthermore, it remains unclear whether such associations might be related to shared familial (genetic and/or environmental) factors that predispose to both preterm birth and diabetes, as opposed to direct effects of preterm birth.

To address these knowledge gaps, we conducted a national cohort study of over 4 million people in Sweden. The goals of this study were to examine associations between gestational age at birth and risk of type 1 or type 2 diabetes up to age 43 years, the maximum follow-up currently possible in this large cohort, to assess whether these associations differ according to sex or fetal growth, and to explore for potential confounding by shared familial (genetic and/or environmental) factors using co-sibling analyses. The results will help inform long-term monitoring, preventive actions and timely detection and treatment of diabetes in the growing population who were born prematurely.

\section{Methods}

Study population The Swedish Birth Registry contains prenatal and birth information for nearly all births nationwide since 1973. Using this registry, we identified all $4,201,706$ singleton live births in Sweden during 1973-2014. We excluded 8637 $(0.2 \%)$ people who had missing information for gestational age, leaving 4,193,069 individuals $(99.8 \%$ of the original cohort) for inclusion in the study. This study was approved by the ethics committee of Lund University in Sweden (No. 
2010/476). Participant consent was not required as this study used only de-identified registry-based secondary data.

Gestational age at birth ascertainment Gestational age at birth was identified from the Swedish Birth Registry based on maternal report of last menstrual period in the 1970s and ultrasonography estimation starting in the 1980s and onward. This was analysed alternatively as a continuous variable or categorical variable with six groups: extremely preterm (22-28 weeks), very preterm (29-33 weeks), late preterm (34-36 weeks), early term (37-38 weeks), fullterm (39-41 weeks, used as the reference group), and post-term ( $\geq 42$ weeks). Early term birth (37-38 weeks) was examined as a separate category because it has previously been associated with increased risk of diabetesrelated mortality relative to later term birth $[6,20]$. In addition, the first three groups were combined to provide summary estimates for preterm birth.

Diabetes ascertainment The study cohort was followed up for the earliest diagnosis of type 1 or type 2 diabetes from birth through the end of follow-up in 2015 (maximum age 43 years; median age at end of follow-up 22.5 years). Type 1 diabetes was defined based on either of the following: (1) any ICD code specific for type 1 diabetes (ICD-9: 250.X1, 250.X3; ICD-10: E10) (for ICD-9 see www.icd9data.com/2007/ Volume1; for ICD-10 see http://apps.who.int/classifications/ icd10/browse/2016/en); or (2) any other ICD code for diabetes (ICD-8/9: 250; ICD-10: E11-E14) combined with insulin prescription (as described below) before age 30 years, consistent with prior epidemiological criteria [21]. Type 2 diabetes was defined based on ICD codes for diabetes (ICD-8/9: 250; ICD-10: E11-E14) that did not meet the additional criteria above for type 1 .

All ICD codes were identified from primary or secondary diagnoses in the Swedish Hospital and Outpatient Registries. The Swedish Hospital Registry was started in 1964 and initially included all hospital discharge diagnoses from the Uppsala region of southern Sweden covering 16\% of the national population, but was expanded to cover nearly $80 \%$ by 1973 (i.e. the beginning of the Swedish Birth Registry and the present study's follow-up period) and $>99 \%$ by 1987 [22, 23]. Diagnoses in this registry have been reported to have a positive predictive value of $\sim 99 \%$ for diabetes [23, 24], although to our knowledge their ability to distinguish type 1 and type 2 has not been specifically evaluated. The Swedish Outpatient Registry contains all outpatient diagnoses from specialty clinics nationwide starting in 2001. The Swedish Pharmacy Registry includes all medication prescriptions nationwide since 1 July 2005, classified according to the Anatomic Therapeutic Chemical (ATC) System. Insulin prescriptions were identified based on any medication prescription with ATC code A10A.
Other study variables Other perinatal and maternal characteristics that may be associated with gestational age at birth and diabetes were identified using the Swedish Birth Registry and national census data, which were linked using an anonymous personal identification number [25-27]. The following were included as adjustment variables: birth year (continuous and categorical by decade), sex, birth order $(1,2, \geq 3)$, maternal age at delivery (continuous), maternal education level $(\leq 9,10-12$, $>12$ years), maternal birth country or region (Sweden, other Europe/US/Canada, Asia/Oceania, Africa, Latin America, other/unknown), maternal BMI (continuous), maternal smoking $(0,1-9, \geq 10$ cigarettes/day), diabetes during pregnancy (i.e. pregestational type 1 or type 2 or gestational diabetes as identified in the birth, hospital or outpatient registries; ICD-8: 250; ICD-9: 250, 648.0, 648.8; ICD-10: E10-E14, O24), preeclampsia (ICD-8: 637; ICD-9: 624.4-624.7; ICD10: O14-O15), and other hypertensive disorders during pregnancy (ICD-8: 400-404; ICD-9: 401-405, 642.0-642.3, 642.9; ICD-10: I10-I15, O10-O11, O13, O15-O16).

Maternal BMI and smoking were assessed at the beginning of prenatal care starting in 1982, and were available for $61.1 \%$ and $74.2 \%$ of participants, respectively. Data were $>99 \%$ complete for all other variables. Missing data for each covariate were imputed using a standard multiple imputation procedure based on the variable's relationship with all other covariates and the outcome [28].

Statistical analysis Cox proportional hazards regression was used to compute HRs and 95\% CIs for associations between gestational age at birth and incident type 1 or type 2 diabetes. These associations were examined from birth to age 43 years and in narrower age intervals ( $<18$ or $18-43$ years) among individuals living in Sweden without a prior diagnosis of the outcome at the beginning of the respective interval. The data were analysed as time-to-event with attained age as the Cox model time axis. To account for competing events that would preclude a diabetes diagnosis, individuals were censored at death as identified in the Swedish Death Registry ( $n=$ 41,$485 ; 1.0 \%$ ) or emigration as determined by absence of a Swedish residential address in census data $(n=258,834$; $6.2 \%$ ). Analyses were conducted both unadjusted and adjusted for covariates (as above). The proportional hazards assumption was assessed by examining log-log plots [29], and was met in each model.

Co-sibling analyses were performed to assess for potential confounding effects of unmeasured shared familial (genetic and/or environmental) factors among all individuals with at least one full sibling $(N=3,481,247 ; 83.0 \%$ of the cohort). This approach can help further elucidate whether associations observed in the primary analyses are due to direct effects of preterm birth as opposed to shared genetic or environmental factors that predispose to both preterm birth and diabetes. Relevant environmental factors within families may 
potentially include lifestyle exposures such as poor diet or physical inactivity. These analyses used stratified Cox regression with a separate stratum for each family as identified by the mother's and father's anonymous identification numbers. In the stratified Cox model, each set of siblings had its own baseline hazard function that reflects the family's shared genetic and environmental factors, and thus associations between gestational age at birth and type 1 or type 2 diabetes were examined within families, controlling for their shared factors. In addition, these analyses were further adjusted for the same covariates as in the main analyses. For type 2 diabetes, co-sibling analyses were also performed after stratifying by sex.

Potential interactions between preterm birth and sex or fetal growth were examined in relation to type 1 or type 2 diabetes risk on the additive and multiplicative scale. Additive interactions were assessed using the 'relative excess risk due to interaction' (RERI), which is computed for binary variables as: $\mathrm{RERI}_{\mathrm{HR}}=\mathrm{HR}_{11}-\mathrm{HR}_{10}-\mathrm{HR}_{01}+1$. Multiplicative interactions were assessed using the ratio of $\mathrm{HRs}: \mathrm{HR}_{11} /\left(\mathrm{HR}_{10} \times\right.$ $\left.\mathrm{HR}_{01}\right)$. A positive additive interaction is indicated if the RERI is $>0$, and a positive multiplicative interaction if the ratio of HRs is $>1[30,31]$.

The following secondary analyses were also performed: (1) Associations were examined between fetal growth (small for gestational age [SGA; $<10$ th percentile], appropriate for gestational age [AGA; 10th-90th percentile], large for gestational age [LGA; $>90$ th percentile]) and type 1 or type 2 diabetes. (2) Type 1 and type 2 diabetes risks were explored after stratifying by spontaneous $(71.4 \%)$ vs medically indicated $(28.6 \%)$ preterm birth, which was systematically recorded starting in $1990(N=2,535,775$ [60.5\% of the cohort]; maximum age 26 years at end of follow-up). (3) As an alternative to multiple imputation, a sensitivity analysis was performed after restricting to participants without missing data $(N=$ 2,561,146 [61.1\% of the cohort]). All statistical tests were 2sided and used an $\alpha$-level of 0.05 . All analyses were conducted using Stata version 15.1 (StataCorp, College Station, TX, USA).

\section{Results}

Table 1 shows perinatal and maternal characteristics by gestational age at birth. Preterm infants were more likely than fullterm infants to be male or firstborn; and their mothers were more likely to be at the extremes of age, have low education level, smoke, or have diabetes, preeclampsia or other hypertensive disorders during their pregnancy.

Associations between gestational age at birth and diabetes In 92.3 million person-years of follow-up, 27,512 (0.7\%) and $5525(0.1 \%)$ individuals were identified with type 1 and type
2 diabetes, respectively. The median age at the end of followup was 22.5 years, and median ages at diagnosis were 14.9 years for type 1 and 29.2 years for type 2 diabetes. The type 1 incidence rate (per 100,000 person-years) was 29.80 in the overall cohort, 36.78 among those born preterm, and 28.80 among those born full-term. The corresponding incidence rates for type 2 diabetes were 5.98, 8.48 and 5.56, respectively.

Gestational age at birth was inversely associated with both type 1 and type 2 diabetes risk at age $<18$ years (adjusted HR per additional week of gestation, type 1: 0.96; $95 \%$ CI 0.95 , 0.97; type 2: 0.95; 0.93, 0.98; Table 2). Adjusted HRs for type 1 and type 2 diabetes associated with preterm birth were 1.21 (95\% CI, 1.14, 1.28) and $1.26(1.01,1.58)$, respectively. In contrast, children born extremely preterm had a lower risk of type 1 diabetes at age $<18$ years (adjusted HR, $0.51 ; 95 \% \mathrm{CI}$ $0.32,0.81$ ) but a near-significantly higher risk of type 2 diabetes $(2.21 ; 95 \% \mathrm{CI}, 0.99,4.95)$, compared with those born fullterm (Table 2).

Gestational age at birth also was inversely associated with new-onset type 1 and type 2 diabetes at age 18-43 years (adjusted HR per additional week of gestation, type 1: 0.96; 95\% CI 0.95, 0.98; type 2: 0.97; 0.95, 0.98). The corresponding HRs comparing preterm vs full-term birth were 1.24 (95\% CI $1.13,1.37)$ and $1.49(1.31,1.68)$, respectively. Extremely preterm birth was associated with $>2$-fold risks of both type 1 (adjusted HR, 2.22; 95\% CI 1.41, 3.48) and type $2(2.55 ; 1.41,4.62)$ diabetes. Preterm birth was more strongly associated with type 2 than type 1 diabetes in adulthood ( $\left.p_{\text {heterogeneity }}<0.001\right)$. Across all models, most adjusted HRs were $<10 \%$ lower than unadjusted HRs (electronic supplementary material [ESM] Table 1). Kaplan-Meier curves for type 1 and type 2 diabetes by gestational age group are shown in Figs. 1 and 2.

Sex-specific analyses Significant interactions were found between preterm birth and sex in relation to type 2 but not type 1 diabetes (ESM Tables 2 and 3). Among individuals born full-term, type 2 diabetes incidence across all attained ages was slightly higher among males (5.84 per 100,000 person-years) than females (5.27). However, the opposite pattern was observed in those born preterm, with higher incidence among females (9.66) than males (7.52) (ESM Table 3). Preterm birth and female sex had a positive interaction on both the additive $(p=0.002)$ and multiplicative $(p=0.006)$ scale (i.e. their combined effect on type 2 diabetes risk was greater than the sum or product of their separate effects; ESM Table 3). The positive additive interaction indicates that preterm birth accounted for significantly more type 2 diabetes cases among females than males. In contrast, no interactions were found between preterm birth and sex in relation to type 1 diabetes on either the additive $(p=0.22)$ or multiplicative $(p=$ 0.09) scale (ESM Table 2). 
Table 1 Characteristics of study participants by gestational age at birth, Sweden, 1973-2014

\begin{tabular}{|c|c|c|c|c|c|c|}
\hline & $\begin{array}{l}\text { Extremely } \\
\text { preterm } \\
(22-28 \text { weeks }) \\
n=11,591 \\
(0.3 \%)\end{array}$ & $\begin{array}{l}\text { Very preterm } \\
(29-33 \text { weeks }) \\
n=41,106 \\
(1.0 \%)\end{array}$ & $\begin{array}{l}\text { Late preterm } \\
(34-36 \text { weeks) } \\
n=157,342 \\
(3.8 \%)\end{array}$ & $\begin{array}{l}\text { Early term } \\
(37-38 \text { weeks }) \\
n=740,391 \\
(17.7 \%)\end{array}$ & $\begin{array}{l}\text { Full-term } \\
(39-41 \text { weeks }) \\
n=2,896,444 \\
(69.1 \%)\end{array}$ & $\begin{array}{l}\text { Post-term } \\
\begin{array}{l}(\geq 42 \text { weeks }) \\
n=346,195 \\
(8.3 \%)\end{array}\end{array}$ \\
\hline \multicolumn{7}{|l|}{ Child characteristics } \\
\hline \multicolumn{7}{|l|}{ Sex } \\
\hline Male & $6288(54.2)$ & $22,975(55.9)$ & $85,570(54.4)$ & $381,140(51.5)$ & $1,471,368(50.8)$ & $188,358(54.4)$ \\
\hline Female & $5303(45.8)$ & $18,131(44.1)$ & $71,772(45.6)$ & $359,251(48.5)$ & $1,425,076(49.2)$ & $157,837(45.6)$ \\
\hline \multicolumn{7}{|l|}{ Fetal growth } \\
\hline SGA & $294(2.5)$ & $5409(13.2)$ & $15,623(9.9)$ & $54,405(7.3)$ & $276,687(9.6)$ & $66,724(19.3)$ \\
\hline AGA & $10,991(94.8)$ & $34,043(82.8)$ & $128,530(81.7)$ & $601,039(81.2)$ & $2,326,431(80.3)$ & $253,586(73.2)$ \\
\hline LGA & $306(2.6)$ & $1654(4.0)$ & $13,189(8.4)$ & $84,947(11.5)$ & $293,326(10.1)$ & $25,885(7.5)$ \\
\hline \multicolumn{7}{|l|}{ Birth order } \\
\hline 1 & 5764 (49.7) & $21,156(51.5)$ & $78,019(49.6)$ & $297,655(40.2)$ & $1,219,056(42.1)$ & $172,702(49.9)$ \\
\hline 2 & 3311 (28.6) & $11,598(28.2)$ & $47,050(29.9)$ & $271,130(36.6)$ & $1,087,624(37.6)$ & $111,055(32.1)$ \\
\hline$\geq 3$ & $2516(21.7)$ & $8352(20.3)$ & $32,273(20.5)$ & $171,606(23.2)$ & $589,764(20.4)$ & $62,438(18.0)$ \\
\hline \multicolumn{7}{|l|}{ Maternal characteristics } \\
\hline \multicolumn{7}{|l|}{ Age (years) } \\
\hline$<20$ & $512(4.4)$ & 1902 (4.6) & $6476(4.1)$ & $22,069(3.0)$ & $84,019(2.9)$ & $12,962(3.7)$ \\
\hline $20-24$ & $2196(18.9)$ & $8336(20.3)$ & $33,190(21.1)$ & $139,115(18.8)$ & $580,841(20.1)$ & $76,287(22.0)$ \\
\hline $25-29$ & $3427(29.6)$ & $12,734(31.0)$ & $51,191(32.5)$ & $243,257(32.9)$ & $1,018,871(35.2)$ & $121,285(35.0)$ \\
\hline $30-34$ & $3148(27.2)$ & $10,954(26.6)$ & $41,555(26.4)$ & $211,859(28.6)$ & $821,668(28.4)$ & $92,813(26.8)$ \\
\hline $35-39$ & $1795(15.5)$ & $5718(13.9)$ & $20,167(12.8)$ & $100,963(13.6)$ & 330,847 (11.4) & $36,883(10.7)$ \\
\hline$\geq 40$ & $513(4.4)$ & $1462(3.6)$ & $4763(3.0)$ & $23,128(3.1)$ & $60,198(2.1)$ & $5965(1.7)$ \\
\hline \multicolumn{7}{|l|}{ Education (years) } \\
\hline$\leq 9$ & 1955 (16.9) & $6768(16.5)$ & $24,405(15.5)$ & $104,171(14.1)$ & $367,829(12.7)$ & $48,594(14.0)$ \\
\hline $10-12$ & $5495(47.4)$ & $19,537(47.5)$ & $74,269(47.2)$ & $338,702(45.7)$ & $1,304,778(45.0)$ & $157,018(45.4)$ \\
\hline$>12$ & $4141(35.7)$ & $14,801(36.0)$ & $58,668(37.3)$ & $297,518(40.2)$ & $1,223,837(42.3)$ & $140,583(40.6)$ \\
\hline \multicolumn{7}{|l|}{ Birth country or region } \\
\hline Sweden & $8984(77.5)$ & 33,666 (81.9) & $130,227(82.8)$ & $603,828(81.6)$ & $2,424,626(83.7)$ & $294,280(85.0)$ \\
\hline $\begin{array}{l}\text { Other Europe/US/ } \\
\text { Canada }\end{array}$ & $1171(10.1)$ & $3581(8.7)$ & $13,239(8.4)$ & $62,313(8.4)$ & $236,119(8.2)$ & $27,999(8.1)$ \\
\hline Asia/Oceania & $826(7.1)$ & $2340(5.7)$ & $9108(5.8)$ & $50,443(6.8)$ & $150,854(5.2)$ & $11,962(3.5)$ \\
\hline Africa & 339 (2.9) & $723(1.8)$ & $2055(1.3)$ & $10,868(1.5)$ & $46,094(1.6)$ & $8025(2.3)$ \\
\hline Latin America & $121(1.0)$ & $360(0.9)$ & $1522(1.0)$ & $8476(1.1)$ & $24,158(0.8)$ & $2014(0.6)$ \\
\hline Other/unknown & $150(1.3)$ & $436(1.1)$ & $1191(0.8)$ & $4463(0.6)$ & $14,593(0.5)$ & $1915(0.6)$ \\
\hline \multicolumn{7}{|l|}{ BMI $\left(\mathrm{kg} / \mathrm{m}^{2}\right)$} \\
\hline$<18.5$ & 217 (1.9) & $1069(2.6)$ & $4808(3.1)$ & $21,784(2.9)$ & $65,600(2.3)$ & $4649(1.3)$ \\
\hline $18.5-24.9$ & $8614(74.3)$ & $31,712(77.2)$ & $121,37(77.1)$ & $567,018(76.6)$ & $2,279,549(78.7)$ & $275,218(79.5)$ \\
\hline $25.0-29.9$ & $1910(16.5)$ & $5735(14.0)$ & $21,663(13.8)$ & $107,959(14.6)$ & $404,340(14.0)$ & $46,600(13.5)$ \\
\hline$\geq 30.0$ & $850(7.3)$ & $2590(6.3)$ & $9534(6.1)$ & $43,630(5.9)$ & $146,991(5.1)$ & $19,728(5.7)$ \\
\hline \multicolumn{7}{|l|}{ Smoking (cigarettes/day) } \\
\hline 0 & $8346(72.0)$ & $29,152(70.9)$ & $114,671(72.9)$ & $565,076(76.3)$ & $2,216,387(76.5)$ & $247,302(71.4)$ \\
\hline $1-9$ & $2557(22.1)$ & $9391(22.8)$ & $33,688(21.4)$ & $138,352(18.7)$ & $567,341(19.6)$ & $87,934(25.4)$ \\
\hline$\geq 10$ & $688(5.9)$ & $2563(6.2)$ & $8983(5.7)$ & $36,963(5.0)$ & $112,716(3.9)$ & $10,959(3.2)$ \\
\hline Diabetes during pregnancy & $336(2.9)$ & $1552(3.8)$ & $6151(3.9)$ & $19,121(2.6)$ & $31,304(1.1)$ & $2260(0.7)$ \\
\hline Preeclampsia & $1650(14.2)$ & $7251(17.6)$ & $16,095(10.2)$ & $39,382(5.3)$ & $94,720(3.3)$ & $11,833(3.4)$ \\
\hline $\begin{array}{l}\text { Other hypertensive } \\
\text { disorders }\end{array}$ & $187(1.6)$ & $714(1.7)$ & $2241(1.4)$ & $8897(1.2)$ & $24,746(0.9)$ & $2347(0.7)$ \\
\hline
\end{tabular}

Data are $n(\%)$

In sex-stratified analyses, preterm birth was associated with significantly increased risks of both type 1 and type 2 diabetes among females and males at all ages, except type 2 diabetes among males at ages $<18$ years (Table 2). Females born preterm had $\sim 1.3$-fold risks and males had $\sim 1$.2-fold risks of type 1 diabetes both at ages $<18$ and $18-43$ years. For type 2 diabetes, adjusted HRs at age $<18$ years were 1.60 (95\% CI $1.18,2.17)$ among females and $1.01(0.73,1.39)$ among males, and at age $18-43$ years were $1.75(1.47,2.09)$ among females and $1.28(1.08,1.53)$ among males. Females born extremely preterm had $>3$-fold risks of type 2 diabetes both at ages $<18$ and $18-43$ years. Figs. 3 and 4 show adjusted HRs for type 1 and type 2 diabetes risk, respectively, by attained age for different gestational age groups.

Co-sibling analyses Co-sibling analyses to control for unmeasured shared familial factors resulted in partial attenuation of most risk estimates (ESM Table 4). At age $<18$ years, the 
Table 2 Associations between gestational age at birth and risk of type 1 and type 2 diabetes, Sweden, 1973-2015

\begin{tabular}{|c|c|c|c|c|c|c|c|c|c|c|}
\hline & \multicolumn{4}{|l|}{ All } & \multicolumn{3}{|c|}{ Females } & \multicolumn{3}{|l|}{ Males } \\
\hline & Cases & Rate $^{\mathrm{a}}$ & $\begin{array}{l}\text { Risk difference } \\
(95 \% \mathrm{CI})^{\mathrm{b}}\end{array}$ & $\begin{array}{l}\text { Adjusted HR } \\
(95 \% \mathrm{CI})^{\mathrm{c}}\end{array}$ & Cases & Rate $^{a}$ & $\begin{array}{l}\text { Adjusted HR } \\
(95 \% \mathrm{CI})^{\mathrm{c}}\end{array}$ & Cases & Rate $^{a}$ & $\begin{array}{l}\text { Adjusted HR } \\
(95 \% \mathrm{CI})^{\mathrm{c}}\end{array}$ \\
\hline \multicolumn{11}{|l|}{ Attained ages $<18$ years } \\
\hline \multicolumn{11}{|l|}{ Type 1 diabetes } \\
\hline Preterm & 1177 & 40.59 & $8.23(5.85,10.61)$ & $1.21(1.14,1.28)$ & 531 & 40.39 & $1.26(1.15,1.38)$ & 646 & 40.75 & $1.17(1.07,1.26)$ \\
\hline Extremely preterm & 18 & 17.33 & $-15.03(-23.05,-7.00)$ & $0.51(0.32,0.81)$ & 7 & 14.16 & $0.44(0.21,0.92)$ & 11 & 20.22 & $0.57(0.32,1.03)$ \\
\hline Very preterm & 172 & 31.52 & $-0.83(-5.58,3.91)$ & $0.93(0.80,1.08)$ & 82 & 33.89 & $1.06(0.85,1.32)$ & 90 & 29.64 & $0.84(0.68,1.04)$ \\
\hline Late preterm & 987 & 43.86 & $11.50(8.71,14.29)$ & $1.30(1.22,1.39)$ & 442 & 43.20 & $1.35(1.22,1.48)$ & 545 & 44.41 & $1.27(1.17,1.39)$ \\
\hline Early term & 4060 & 38.31 & $5.95(4.65,7.25)$ & $1.16(1.12,1.20)$ & 1813 & 35.40 & $1.12(1.06,1.18)$ & 2247 & 41.04 & $1.19(1.14,1.25)$ \\
\hline Full-term & 13,610 & 32.36 & Reference & Reference & 6360 & 30.72 & Reference & 7250 & 33.95 & Reference \\
\hline Post-term & 1406 & 27.05 & $-5.31(-6.82,-3.79)$ & $0.86(0.81,0.91)$ & 617 & 25.68 & $0.87(0.80,0.95)$ & 789 & 28.24 & $0.85(0.79,0.91)$ \\
\hline $\begin{array}{l}\text { Per additional } \\
\text { week (trend) }\end{array}$ & & & & $0.96(0.95,0.97)$ & & & $0.96(0.95,0.97)$ & & & $0.96(0.95,0.97)$ \\
\hline \multicolumn{11}{|l|}{ Type 2 diabetes } \\
\hline Preterm & 89 & 3.07 & $0.93(0.27,1.58)$ & $1.26(1.01,1.58)$ & 48 & 3.65 & $1.60(1.18,2.17)$ & 41 & 2.59 & $1.01(0.73,1.39)$ \\
\hline Extremely preterm & 6 & 5.78 & $3.63(-0.99,8.26)$ & $2.21(0.99,4.95)$ & 4 & 8.09 & $3.38(1.26,9.06)$ & 2 & 3.68 & $1.32(0.33,5.28)$ \\
\hline Very preterm & 25 & 4.58 & $2.44(-0.64,4.24)$ & $1.85(1.24,2.77)$ & 13 & 5.37 & $2.33(1.33,4.07)$ & 12 & 3.95 & $1.50(0.84,2.67)$ \\
\hline Late preterm & 58 & 2.58 & $0.44(-0.24,1.11)$ & $1.07(0.82,1.40)$ & 31 & 3.03 & $1.34(0.92,1.93)$ & 27 & 2.20 & $0.87(0.59,1.28)$ \\
\hline Early term & 328 & 3.10 & $0.95(0.59,1.32)$ & $1.33(1.17,1.51)$ & 177 & 3.46 & $1.56(1.31,1.86)$ & 151 & 2.76 & $1.13(0.94,1.36)$ \\
\hline Full-term & 901 & 2.14 & Reference & Reference & 421 & 2.03 & Reference & 480 & 2.25 & Reference \\
\hline Post-term & 127 & 2.44 & $0.30(-0.15,0.75)$ & $1.20(0.99,1.45)$ & 46 & 1.91 & $1.02(0.75,1.38)$ & 81 & 2.90 & $1.33(1.05,1.68)$ \\
\hline $\begin{array}{l}\text { Per additional week } \\
\text { (trend) }\end{array}$ & & & & $0.95(0.93,0.98)$ & & & $0.91(0.88,0.94)$ & & & $0.99(0.96,1.03)$ \\
\hline \multicolumn{11}{|l|}{ Attained ages $18-43$ years } \\
\hline \multicolumn{11}{|l|}{ Type 1 diabetes } \\
\hline Preterm & 427 & 29.22 & $7.30(4.46,10.14)$ & $1.24(1.13,1.37)$ & 168 & 25.77 & $1.34(1.14,1.57)$ & 259 & 32.00 & $1.19(1.05,1.35)$ \\
\hline Extremely preterm & 19 & 49.66 & $27.74(5.40,50.08)$ & $2.22(1.41,3.48)$ & 12 & 64.48 & $3.55(2.01,6.26)$ & 7 & 35.62 & $1.35(0.64,2.84)$ \\
\hline Very preterm & 78 & 29.25 & $7.33(0.81,13.85)$ & $1.25(1.00,1.56)$ & 28 & 23.89 & $1.24(0.85,1.80)$ & 50 & 33.46 & $1.25(0.95,1.66)$ \\
\hline Late preterm & 330 & 28.54 & $6.62(3.47,9.76)$ & $1.21(1.08,1.36)$ & 128 & 24.80 & $1.28(1.07,1.53)$ & 202 & 31.55 & $1.17(1.01,1.35)$ \\
\hline Early term & 1349 & 26.46 & $4.54(3.00,6.08)$ & $1.17(1.10,1.24)$ & 511 & 21.39 & $1.14(1.04,1.26)$ & 838 & 30.93 & $1.18(1.09,1.28)$ \\
\hline Full-term & 4773 & 21.92 & Reference & Reference & 1934 & 18.11 & Reference & 2839 & 25.59 & Reference \\
\hline Post-term & 710 & 21.93 & $0.01(-1.72,1.74)$ & $1.00(0.93,1.08)$ & 277 & 17.66 & $0.98(0.86,1.11)$ & 433 & 25.96 & $1.02(0.92,1.12)$ \\
\hline $\begin{array}{l}\text { Per additional } \\
\text { week (trend) }\end{array}$ & & & & $0.96(0.95,0.98)$ & & & $0.96(0.94,0.98)$ & & & $0.97(0.95,0.98)$ \\
\hline \multicolumn{11}{|l|}{ Type 2 diabetes } \\
\hline Preterm & 281 & 19.23 & $7.08(4.78,9.37)$ & $1.49(1.31,1.68)$ & 142 & 21.78 & $1.75(1.47,2.09)$ & 139 & 17.17 & $1.28(1.08,1.53)$ \\
\hline Extremely preterm & 11 & 28.75 & $16.60(-0.40,33.60)$ & $2.55(1.41,4.62)$ & 7 & 37.61 & $3.50(1.67,7.37)$ & 4 & 20.36 & $1.74(0.65,4.63)$ \\
\hline Very preterm & 49 & 18.38 & $6.22(1.06,11.39)$ & $1.42(1.07,1.88)$ & 30 & 25.60 & $2.05(1.43,2.95)$ & 19 & 12.71 & $0.95(0.60,1.49)$ \\
\hline Late preterm & 221 & 19.11 & $6.96(4.40,9.52)$ & $1.47(1.28,1.69)$ & 105 & 20.34 & $1.63(1.33,1.99)$ & 116 & 18.12 & $1.35(1.12,1.63)$ \\
\hline Early term & 656 & 12.87 & $0.72(-0.37,1.80)$ & $1.09(1.00,1.18)$ & 308 & 12.89 & $1.14(1.01,1.30)$ & 348 & 12.84 & $1.04(0.92,1.17)$ \\
\hline Full-term & 2646 & 12.15 & Reference & Reference & 1232 & 11.54 & Reference & 1414 & 12.74 & Reference \\
\hline Post-term & 497 & 15.35 & $3.20(1.77,4.63)$ & $1.10(1.00,1.21)$ & 211 & 13.45 & $1.02(0.88,1.18)$ & 286 & 17.14 & $1.17(1.03,1.33)$ \\
\hline $\begin{array}{l}\text { Per additional } \\
\text { week (trend) }\end{array}$ & & & & $0.97(0.95,0.98)$ & & & $0.94(0.92,0.96)$ & & & $0.99(0.97,1.01)$ \\
\hline
\end{tabular}

${ }^{\mathrm{a}}$ Incidence rate per 100,000 person-years

${ }^{\mathrm{b}}$ Incidence rate difference per 100,000 person-years

${ }^{\mathrm{c}}$ Adjusted for child characteristics (birth year, sex, birth order) and maternal characteristics (age, education, birth country or region, BMI, smoking, diabetes, preeclampsia, other hypertensive disorders during pregnancy)

Preterm, <37 weeks; extremely preterm, 22-28 weeks; very preterm, 29-33 weeks; late preterm, 34-36 weeks; early term, 37-38 weeks; full-term, 3941 weeks; post-term, $\geq 42$ weeks)

adjusted HR for type 1 diabetes associated with preterm birth was $1.21(95 \% \mathrm{CI} 1.14,1.28)$ in the primary analysis and $1.16(1.04,1.30)$ in the co-sibling analysis; the corresponding HRs for type 2 diabetes were 1.26 (1.01, $1.58)$ and $1.13(0.75,1.70$; based on only 21 cases $)$, respectively. At age 18-43 years, the adjusted HR for type 1 diabetes associated with preterm birth was 1.24 (95\% CI 1.13, 1.37) in the primary analysis and 1.14 $(0.92,1.41)$ in the co-sibling analysis. In contrast, the corresponding $\mathrm{HR}$ for type 2 diabetes at age 18- 


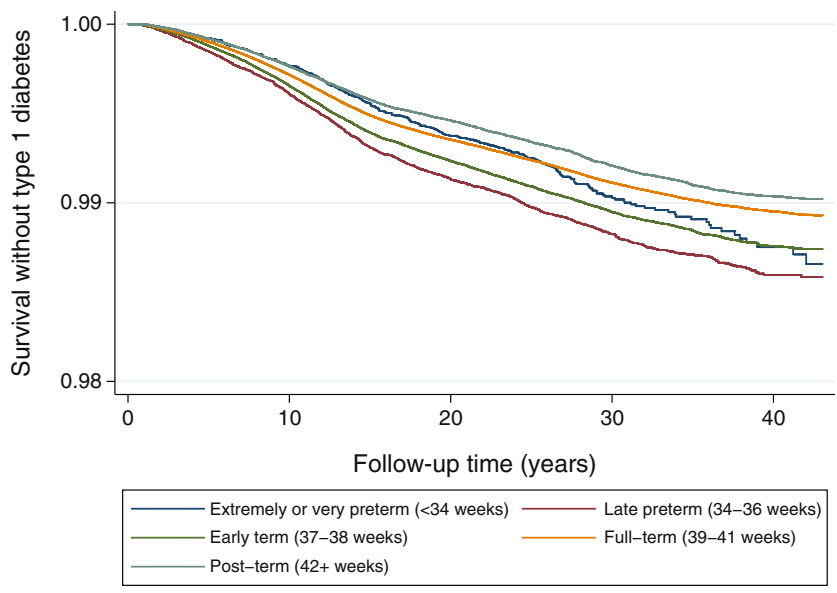

Fig. 1 Kaplan-Meier curves for time to type 1 diabetes

43 years in the primary analysis $(1.49 ; 95 \%$ CI 1.31 , 1.68) was not attenuated in the co-sibling analysis $(1.55 ; 1.12,2.13)$.

After stratifying by sex, co-sibling analyses of type 2 diabetes yielded similar results. For example, the adjusted HR at age 18-43 years comparing preterm with full-term birth was $1.50(95 \%$ CI $0.78,2.86)$ among full sisters and $1.41(0.75$, 2.64) among full brothers.

Secondary analyses In analyses of fetal growth, SGA was a strong risk factor for type 2 but not type 1 diabetes, compared with AGA (e.g. age < 18 years, type 1: adjusted HR, $0.88 ; 95 \%$ CI $0.83,0.93$; type $2: 1.61 ; 1.38,1.89$; age $18-43$ years, type 1: $1.17 ; 1.09,1.26$; type $2: 1.79 ; 1.65,1.93$; see ESM Table 5 for complete results). The risk of type 2 diabetes was highest among individuals born both preterm and SGA (adjusted HR, 2.24; $95 \%$ CI 1.70, 2.96; relative to those born full-term and AGA). However, there was no evidence of interaction between preterm birth and SGA in relation to type 1 or type 2 diabetes risk on either the additive or multiplicative scale (type $1: p=0.13$ and $p=0.13$; type $2: p=0.81$ and $p=0.52$, respectively; ESM Tables 6 and 7). In a sensitivity analysis

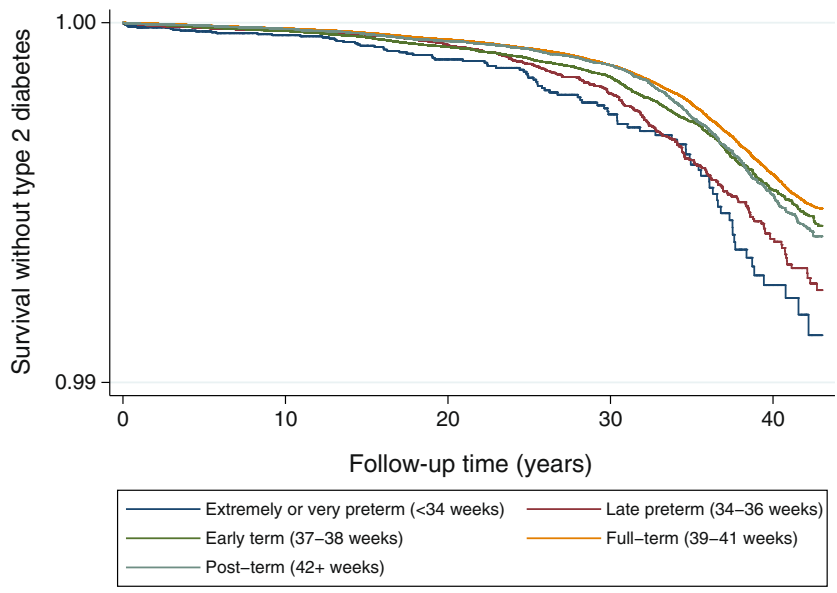

Fig. 2 Kaplan-Meier curves for time to type 2 diabetes that was restricted to AGA births, all results were negligibly changed (e.g. preterm vs full-term, ages $<18$ years, type 1 : adjusted HR, 1.20; 95\% CI 1.12, 1.28; type 2: 1.24; 0.96, 1.60 ; ages $18-43$ years, type $1: 1.24 ; 1.11,1.39$; type 2 : $1.49 ; 1.29,1.72$ ).

Compared with full-term birth, both spontaneous and medically indicated preterm birth were associated with increased risks of type 1 diabetes (adjusted HR, 1.22; 95\% CI 1.11, 1.34; and 1.22; 1.09, 1.37, respectively; $p=0.97$ for difference in HRs) and type 2 diabetes $(1.41 ; 1.05,1.90$; and $1.45 ; 1.02,2.04$, respectively; $p=0.92$ for difference in HRs).

Restricting to participants without missing data resulted in modest changes in most risk estimates (e.g. preterm vs fullterm, age < 18 years, type 1 diabetes: adjusted HR, 1.22; 95\% CI 1.13, 1.32; type 2 diabetes: $1.42 ; 1.08,1.87$; age 18 43 years, type $1: 1.07 ; 0.87,1.31$; type $2: 1.96 ; 1.48,2.61$ ).

\section{Discussion}

In this large national cohort study, preterm birth was associated with increased risks of type 1 and type 2 diabetes from childhood into early to mid-adulthood. Preterm birth was associated with approximately 1.2- and 1.3-fold risk of type 1 and type 2 diabetes, respectively, at age $<18$ years, and 1.2and 1.5 -fold risk, respectively, at age $18-43$ years. The associations between preterm birth and type 2 (but not type 1) diabetes were significantly stronger among females. Early term birth (37-38 weeks) was also associated with modestly increased risk of type 1 and type 2 diabetes from childhood into adulthood. Co-sibling analyses suggested that these findings were only partially explained by shared genetic or environmental factors in families.

Several prior studies have linked preterm birth with type 1 diabetes in childhood. A Swedish cohort study of 3.6 million children aged $<15$ years, who overlapped with the present cohort, reported 10-20\% increased risk of type 1 diabetes among those born at 33-36 or 37-38 weeks [11]. However, consistent with our findings, infants born at $<33$ weeks had lower risk of type 1 diabetes during childhood compared with those born at term. To our knowledge, this finding remains unexplained and has not been assessed in other studies, and thus will need confirmation in other large independent cohorts. A UK cohort study of 3.8 million children aged $<12$ years reported $15-30 \%$ increased risk of type 1 diabetes among those born preterm or early term compared with fullterm, but did not specifically examine earlier gestational ages [10]. An Australian cohort study of 558,633 children aged $<15$ years reported 1.4- and 1.2-fold risk among those born preterm or early term, respectively [13]. A matched cohort study in Taiwan with 37,119 preterm and 162,020 term children (mean age 8-9 years) reported 1.8- and 2.5-fold risks of type 1 and type 2 diabetes, respectively [14]. A Swedish 
Fig. 3 Adjusted HRs for newonset type 1 diabetes by gestational age at birth compared with full-term birth, Sweden, 1973-2015

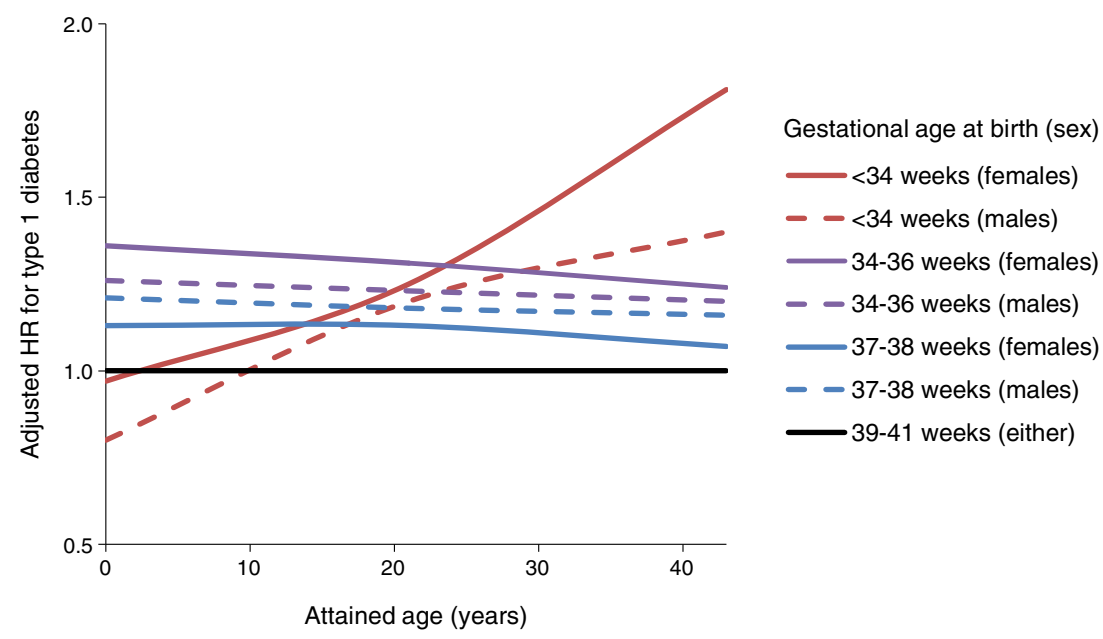

cohort study of 630,090 adults aged 25-37 years found that those born preterm had modestly (10-25\%) increased odds of medication prescription for diabetes, which was predominantly type 1 [32].

Several smaller studies have also reported associations between preterm birth and type 2 diabetes in mid-adulthood. For example, a Finnish cohort study of 12,813 adults aged $>40$ years reported a 1.6 -fold $(95 \%$ CI $1.00,2.52)$ risk for those born at gestational age $<35$ vs $37-41$ weeks [16]. A Swedish cohort study of 6425 adults aged $37-62$ years reported that those born at $<33$ weeks had a 1.6-fold (95\% CI 1.33, 2.11) risk of type 2 diabetes based on inpatient diagnoses [17]. A Scottish cohort study of 5973 adults aged 46-50 years found that preterm birth was associated with a 2-fold $(95 \%$ CI 1.18, 3.53) risk of self-reported type 2 diabetes [18]. In the largest meta-analysis to date with $\sim 2.2$ million participants from 23 studies, the pooled ORs for association between preterm birth and type 1 or type 2 diabetes were 1.18 (95\% CI $1.11,1.25$; based on 18 studies) and $1.51(1.32,1.72 ; 5$ studies), respectively [33].
To our knowledge, no prior studies have examined gestational age at birth in relation to both type 1 and type 2 diabetes and potential sex-specific differences from childhood into adulthood. The present study addressed these gaps using nationwide diagnoses in the largest cohort to date, while controlling for multiple potential confounders. In this cohort and in other general populations, type 2 diabetes has a higher overall prevalence among men $[1,2]$. However, we found that preterm birth was more strongly associated with type 2 diabetes and accounted for significantly more cases among women. To our knowledge, this sex-specific difference has not been previously reported and thus warrants confirmation in other well-powered studies. We found that most of the observed associations were partially explained by shared genetic or environmental factors in families. However, the association between preterm birth and type 2 diabetes in adulthood specifically appeared independent of shared familial factors.

These findings may have multiple underlying mechanisms that involve pancreatic beta cell function and insulin resistance. Preterm birth interrupts the development of pancreatic
Fig. 4 Adjusted HRs for newonset type 2 diabetes by gestational age at birth compared with full-term birth, Sweden, 1973-2015

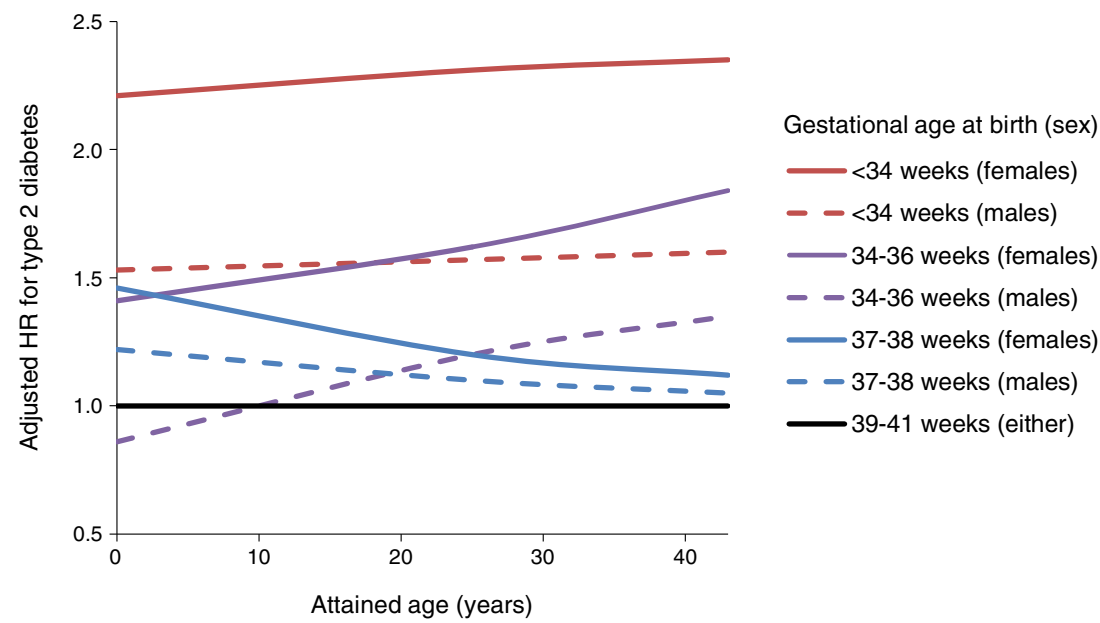


beta cells, which are formed predominantly in the third trimester of pregnancy, and might permanently reduce their number or function [34]. The limited available evidence for beta cell mass and function after preterm birth is conflicting. For example, experimental evidence has shown that induced preterm birth in sheep resulted in a $65 \%$ reduction of beta cell mass and reduced insulin secretory capacity that persisted into adulthood [35]. However, limited evidence from human studies has suggested that prematurity may be associated with appropriate insulin secretion consistent with normal beta cell function in early or mid-adulthood [36,37]. Preterm birth also alters immune function including T cell response [38], which may potentially mediate its association with type 1 diabetes, consistent with its autoimmune aetiology [39]. Other contributing factors may include exposure to antenatal corticosteroids and rapid catch-up growth in infancy, leading to visceral adiposity and insulin resistance [40-42]. Iatrogenic factors from intensive care, including suboptimal nutrition and adverse effects of medications or procedures, may further impair glucose metabolism [4]. Several studies have reported reduced insulin sensitivity in preterm-born children or adults compared with term-born controls [42-44]. These associations are further modified by lifestyle factors across the life course, including diet, exercise and obesity [45], which are important targets for intervention.

Because of major advances in neonatal and paediatric care, most preterm infants now survive into adulthood [6]. As a result, clinicians will increasingly encounter adult patients who were born prematurely. Preterm birth should now be recognised as a chronic condition that predisposes to the development of diabetes across the life course. Physicians currently seldom seek birth histories from adult patients, and thus preterm birth may remain a 'hidden' risk factor. Medical records and history-taking in patients of all ages should routinely include birth history, including gestational age, birthweight and perinatal complications [4, 46-48]. Such information can help identify those born prematurely and facilitate anticipatory screening and early preventive actions, including patient counselling to promote lifestyle prevention of diabetes.

A key strength of the present study was the ability to examine gestational age at birth in relation to both type 1 and type 2 diabetes in a large national cohort with follow-up into adulthood, using birth, medical and pharmacy registry data that are highly complete. This study design minimises potential selection or ascertainment biases and enables more robust risk estimates based on a national population. The large sample size enabled well-powered assessment of narrowly defined gestational age groups and sex-specific differences. The results were controlled for other perinatal and maternal factors, as well as unmeasured familial factors using co-sibling analyses.
This study also had several limitations. First, laboratory data to verify diagnoses were unavailable. High positive predictive values have been reported for most chronic disorders in the Swedish registries, including diabetes ( $>99 \%)[23$, 24]. However, to our knowledge, the validity of registry diagnoses for distinguishing type 1 and type 2 diabetes has not been evaluated. Our observed incidences were slightly lower than those previously reported based on serologic testing of autoantibodies and C-peptide to distinguish type 1 from type 2 diabetes in 1630 Swedish individuals of similar ages (e.g. type 1 : 37.8 per 100,000 person-years at age $0-19$ years vs 33.3 at age $0-18$ years in the present study; type 2: 3.1 per 100,000 person-years at age $0-19$ years vs 2.4 at age $0-18$ years in the present study) [49]. It is possible that people born prematurely are more likely to be diagnosed with diabetes because of greater contact with the healthcare system (i.e. detection bias). However, this is most likely to affect relatively asymptomatic conditions early in life. Detection bias is less likely for type 1 diabetes because it is highly symptomatic, or in adulthood when most type 2 diabetes is diagnosed. Second, despite up to 43 years of follow-up, this was still a relatively young cohort. Additional follow-up will be needed to examine diabetes risks in older adulthood when such data become available in this or other large cohorts. Finally, this study was limited to Sweden and will need replication in other countries and diverse populations.

In summary, we found that preterm and early term birth were associated with increased risk of type 1 and type 2 diabetes from childhood into early to mid-adulthood in a large population-based cohort. Children and adults who were born prematurely may need early preventive evaluation and longterm follow-up for timely detection and treatment of diabetes.

Acknowledgements Open access funding provided by Lund University.

Data availability Owing to ethical concerns, supporting data cannot be made openly available. Further information about the data registries is available from the Swedish National Board of Health and Welfare: https:// www.socialstyrelsen.se/en/statistics-and-data/registers/.

Funding This work was supported by the National Heart, Lung, and Blood Institute at the National Institutes of Health [R01 HL139536 to $\mathrm{CC}$ and KS]; the Swedish Research Council; the Swedish Heart-Lung Foundation; and ALF project grant, Region Skåne/Lund University, Sweden. The study sponsors were not involved in the design of the study; the collection, analysis, and interpretation of data; writing the report; or the decision to submit the report for publication.

Duality of interest The authors declare that there is no duality of interest associated with this manuscript.

Contribution statement $\mathrm{CC}$, JS and $\mathrm{KS}$ conceived and designed the study and obtained funding. JS and KS acquired data and all authors analysed and interpreted the data. CC drafted the manuscript and all authors critically revised it for important intellectual content. All authors gave final approval of the version to be published. JS is the guarantor and, as such, had full access to all of the data in the study and takes responsibility for the integrity of the data and the accuracy of the data analysis. 
Open Access This article is distributed under the terms of the Creative Commons Attribution 4.0 International License (http:// creativecommons.org/licenses/by/4.0/), which permits unrestricted use, distribution, and reproduction in any medium, provided you give appropriate credit to the original author(s) and the source, provide a link to the Creative Commons license, and indicate if changes were made.

\section{References}

1. Xu G, Liu B, Sun Y et al (2018) Prevalence of diagnosed type 1 and type 2 diabetes among US adults in 2016 and 2017: population based study. BMJ 362:k1497. https://doi.org/10.1136/bmj.k1497

2. Cho NH, Shaw JE, Karuranga S et al (2018) IDF Diabetes Atlas: global estimates of diabetes prevalence for 2017 and projections for 2045. Diabetes Res Clin Pract 138:271-281. https://doi.org/10. 1016/j.diabres.2018.02.023

3. Barker DJ (2005) The developmental origins of insulin resistance. Horm Res 64(Suppl 3):2-7. https://doi.org/10.1159/000089311

4. Raju TNK, Buist AS, Blaisdell CJ, Moxey-Mims M, Saigal S (2017) Adults born preterm: a review of general health and system-specific outcomes. Acta Paediatr 106(9):1409-1437. https://doi.org/10.1111/apa.13880

5. Crump C, Sundquist K, Sundquist J, Winkleby MA (2011) Gestational age at birth and mortality in young adulthood. JAMA 306(11):1233-1240. https://doi.org/10.1001/jama.2011.1331

6. Crump C, Sundquist J, Winkleby MA, Sundquist K (2019) Gestational age at birth and mortality from infancy into mid-adulthood: a national cohort study. Lancet Child Adolesc Health 3(6): 408-417. https://doi.org/10.1016/S2352-4642(19)30108-7

7. Blencowe H, Cousens S, Oestergaard MZ et al (2012) National, regional, and worldwide estimates of preterm birth rates in the year 2010 with time trends since 1990 for selected countries: a systematic analysis and implications. Lancet 379(9832):2162-2172. https://doi.org/10.1016/S0140-6736(12)60820-4

8. Purisch SE, Gyamfi-Bannerman C (2017) Epidemiology of preterm birth. Semin Perinatol 41(7):387-391. https://doi.org/10.1053/j. semperi.2017.07.009

9. March of Dimes (2019) PeriStats. Available from http://www. marchofdimes.com/Peristats/. Accessed 1 April 2019

10. Goldacre RR (2018) Associations between birthweight, gestational age at birth and subsequent type 1 diabetes in children under 12: a retrospective cohort study in England, 1998-2012. Diabetologia 61(3):616-625. https://doi.org/10.1007/s00125-017-4493-y

11. Khashan AS, Kenny LC, Lundholm C et al (2015) Gestational age and birth weight and the risk of childhood type 1 diabetes: a population-based cohort and sibling design study. Diabetes Care 38(12):2308-2315. https://doi.org/10.2337/dc15-0897

12. Cardwell CR, Carson DJ, Patterson CC (2005) Parental age at delivery, birth order, birth weight and gestational age are associated with the risk of childhood type 1 diabetes: a UK regional retrospective cohort study. Diabet Med 22(2):200-206. https://doi.org/10. 1111/j.1464-5491.2005.01369.x

13. Haynes A, Bower C, Bulsara MK, Finn J, Jones TW, Davis EA (2007) Perinatal risk factors for childhood type 1 diabetes in Western Australia-a population-based study (1980-2002). Diabet Med 24(5):564-570. https://doi.org/10.1111/j.1464-5491.2007. 02149.X

14. Huang YT, Lin HY, Wang CH, Su BH, Lin CC (2018) Association of preterm birth and small for gestational age with metabolic outcomes in children and adolescents: a population-based cohort study from Taiwan. Pediatr Neonatol 59(2):147-153. https://doi. org/10.1016/j.pedneo.2017.07.007

15. Algert CS, McElduff A, Morris JM, Roberts CL (2009) Perinatal risk factors for early onset of type 1 diabetes in a 2000-2005 birth cohort. Diabet Med 26(12):1193-1197. https://doi.org/10.1111/j. 1464-5491.2009.02878.x

16. Kajantie E, Osmond C, Barker DJ, Eriksson JG (2010) Preterm birth-a risk factor for type 2 diabetes? The Helsinki birth cohort study. Diabetes Care 33(12):2623-2625. https://doi.org/10.2337/ dc10-0912

17. Kaijser M, Bonamy AK, Akre O et al (2009) Perinatal risk factors for diabetes in later life. Diabetes 58(3):523-526. https://doi.org/10. 2337/db08-0558

18. Lawlor DA, Davey Smith G, Clark H, Leon DA (2006) The associations of birthweight, gestational age and childhood BMI with type 2 diabetes: findings from the Aberdeen children of the 1950s cohort. Diabetologia 49(11):2614-2617. https://doi.org/10.1007/ s00125-006-0408-Z

19. Pilgaard K, Faerch K, Carstensen B et al (2010) Low birthweight and premature birth are both associated with type 2 diabetes in a random sample of middle-aged Danes. Diabetologia 53(12):25262530. https://doi.org/10.1007/s00125-010-1917-3

20. Crump C, Sundquist K, Winkleby MA, Sundquist J (2013) Earlyterm birth (37-38 weeks) and mortality in young adulthood. Epidemiology 24(2):270-276. https://doi.org/10.1097/EDE. 0b013e318280da0f

21. Rawshani A, Rawshani A, Franzen S et al (2017) Mortality and cardiovascular disease in type 1 and type 2 diabetes. $\mathrm{N}$ Engl J Med 376(15):1407-1418. https://doi.org/10.1056/ NEJMoa1608664

22. Swedish National Board of Health and Welfare (2019) The National Patient Register. Available from https://www.socialstyrelsen.se/en/ statistics-and-data/registers/alla-register/the-national-patientregister/. Accessed 3 September 2019

23. Ludvigsson JF, Andersson E, Ekbom A et al (2011) External review and validation of the Swedish national inpatient register. BMC Public Health 11:450. https://doi.org/10.1186/1471-2458-11-450

24. Ragnarson Tennvall G, Apelqvist J, Eneroth M (2000) The inpatient care of patients with diabetes mellitus and foot ulcers. A validation study of the correspondence between medical records and the Swedish Inpatient Registry with the consequences for cost estimations. J Intern Med 248(5):397-405

25. Crump C, Howell EA, Stroustrup A, McLaughlin MA, Sundquist J, Sundquist K (2019) Association of preterm birth with risk of ischemic heart disease in adulthood. JAMA Pediatr. https://doi.org/10. 1001/jamapediatrics.2019.1327

26. Crump C, Sundquist J, Winkleby MA, Sundquist K (2019) Preterm birth and risk of chronic kidney disease from childhood into midadulthood: national cohort study. BMJ 365:11346. https://doi.org/ 10.1136/bmj.11346

27. Crump C, Friberg D, Li X, Sundquist J, Sundquist K (2019) Preterm birth and risk of sleep-disordered breathing from childhood into mid-adulthood. Int J Epidemiol pii: dyz075. https://doi.org/10. 1093/ije/dyz075

28. Rubin DB (1987) Multiple imputation for nonresponse in surveys. Wiley, New York

29. Grambsch PM (1995) Goodness-of-fit and diagnostics for proportional hazards regression models. Cancer Treat Res 75:95-112

30. Li R, Chambless L (2007) Test for additive interaction in proportional hazards models. Ann Epidemiol 17(3):227-236. https://doi. org/10.1016/j.annepidem.2006.10.009

31. VanderWeele TJ (2011) Causal interactions in the proportional hazards model. Epidemiology 22(5):713-717. https://doi.org/10. 1097/EDE.0b013e31821db503

32. Crump C, Winkleby MA, Sundquist K, Sundquist J (2011) Risk of diabetes among young adults born preterm in Sweden. Diabetes Care 34(5):1109-1113. https://doi.org/10.2337/dc10-2108

33. Li S, Zhang M, Tian H, Liu Z, Yin X, Xi B (2014) Preterm birth and risk of type 1 and type 2 diabetes: systematic review and meta- 
analysis. Obes Rev 15(10):804-811. https://doi.org/10.1111/obr. 12214

34. Gregg BE, Moore PC, Demozay D et al (2012) Formation of a human beta-cell population within pancreatic islets is set early in life. J Clin Endocrinol Metab 97(9):3197-3206. https://doi.org/10. 1210/jc.2012-1206

35. Bansal A, Bloomfield FH, Connor KL et al (2015) Glucocorticoidinduced preterm birth and neonatal hyperglycemia alter ovine $\beta$ cell development. Endocrinology 156(10):3763-3776. https://doi. org/10.1210/en.2015-1095

36. Mathai S, Cutfield WS, Derraik JG et al (2012) Insulin sensitivity and $\beta$-cell function in adults born preterm and their children. Diabetes 61(10):2479-2483. https://doi.org/10.2337/db11-1672

37. Pilgaard K, Faerch K, Poulsen P et al (2010) Impact of size at birth and prematurity on adult anthropometry in 4744 middle-aged Danes - the Inter99 study. J Dev Orig Health Dis 1(5):319-328. https://doi.org/10.1017/S2040174410000413

38. Sharma AA, Jen R, Butler A, Lavoie PM (2012) The developing human preterm neonatal immune system: a case for more research in this area. Clin Immunol 145(1):61-68. https://doi.org/10.1016/j. clim.2012.08.006

39. Bloomfield FH (2018) Impact of prematurity for pancreatic islet and beta-cell development. J Endocrinol 238(3):R161-R171. https://doi.org/10.1530/JOE-18-0021

40. Kajantie E, Strang-Karlsson S, Hovi P et al (2015) Insulin sensitivity and secretory response in adults born preterm: the Helsinki study of very low birth weight adults. J Clin Endocrinol Metab 100(1): 244-250. https://doi.org/10.1210/jc.2014-3184

41. Parkinson JR, Hyde MJ, Gale C, Santhakumaran S, Modi N (2013) Preterm birth and the metabolic syndrome in adult life: a systematic review and meta-analysis. Pediatrics 131(4):e1240-e1263. https:// doi.org/10.1542/peds.2012-2177
42. Hofman PL, Regan F, Jackson WE et al (2004) Premature birth and later insulin resistance. N Engl J Med 351(21):2179-2186. https:// doi.org/10.1056/NEJMoa042275

43. Hovi P, Andersson S, Eriksson JG et al (2007) Glucose regulation in young adults with very low birth weight. N Engl J Med 356(20): 2053-2063. https://doi.org/10.1056/NEJMoa067187

44. Rotteveel J, van Weissenbruch MM, Twisk JW, Delemarre-Van de Waal HA (2008) Infant and childhood growth patterns, insulin sensitivity, and blood pressure in prematurely born young adults. Pediatrics 122(2):313-321. https://doi.org/10.1542/peds.20072012

45. Tinnion R, Gillone J, Cheetham T, Embleton N (2014) Preterm birth and subsequent insulin sensitivity: a systematic review. Arch Dis Child 99(4):362-368. https://doi.org/10.1136/archdischild2013-304615

46. Crump C (2014) Medical history taking in adults should include questions about preterm birth. BMJ 349:g4860. https://doi.org/10. 1136/bmj.g4860

47. Crump C (2015) Birth history is forever: implications for family medicine. J Am Board Fam Med 28(1):121-123. https://doi.org/10. 3122/jabfm.2015.01.130317

48. Crump C, Sundquist K, Sundquist J (2016) Adult outcomes of preterm birth. Prev Med 91:400-401. https://doi.org/10.1016/j. ypmed.2016.08.024

49. Thunander M, Petersson C, Jonzon K et al (2008) Incidence of type 1 and type 2 diabetes in adults and children in Kronoberg, Sweden. Diabetes Res Clin Pract 82(2):247-255. https://doi.org/10.1016/j. diabres.2008.07.022

Publisher's note Springer Nature remains neutral with regard to jurisdictional claims in published maps and institutional affiliations. 